\title{
The spectral analysis of photoplethysmography to evaluate an independent cardiovascular risk factor
}

This article was published in the following Dove Press journal:

International Journal of General Medicine

9 December 2014

Number of times this article has been viewed

\author{
Pratiksha G Gandhi' \\ Gundu HR Rao² \\ IIPC Heart Care Centre, Mumbai, \\ India; ' University of Minnesota, \\ Minneapolis, MN, USA
}

Correspondence: Pratiksha G Gandhi Tel +9l 9022530033 (India) Fax +I 9094509625 (US)

Email chairpersonipc@yahoo.co.in
Background: In this study, we evaluate homeostatic markers correlated to autonomic nervous and endothelial functions in a population of coronary artery disease (CAD) patients versus a control group. Since CAD is the highest risk marker for sudden cardiac death, the study objective is to determine whether an independent cardiovascular risk score based on these markers can be used alongside known conventional cardiovascular risk markers to strengthen the understanding of a patient's vascular state.

Materials and methods: Sixty-five subjects (13 women) with a mean age of 62.9 years (range 40-80 years) who were diagnosed with CAD using coronary angiography (group 1) and seventy-two subjects ( 29 women) with a mean age of 45.1 years (range 18-85 years) who claimed they were healthy (group 2) were included in the study. These subjects underwent examination with the TM-Oxi and SudoPath systems at IPC Heart Care Centers in Mumbai, India. The TM-Oxi system takes measurements from a blood pressure device and a pulse oximeter. The SudoPath measures galvanic skin response to assess the sudomotor pathway function. Spectral analysis of the photoplethysmograph (PTG) waveform and electrochemical galvanic skin response allow the TM-Oxi and SudoPath systems to calculate several homeostatic markers, such as the PTG index (PTGi), PTG very low frequency index (PTGVLFi), and PTG ratio (PTGr). The focus of this study was to evaluate these markers (PTGi, PTGVLFi, and PTGr) in CAD patients against a control group, and to calculate an independent cardiovascular risk factor score: the PTG cardiovascular disease risk score (PTG CVD), calculated solely from these markers. We compared PTGi, PTGVLFi, PTGr, and PTG CVD scores between the CAD patient group and the healthy control group. Statistical analyses were performed using receiver operating characteristic curves to determine the specificity and sensitivity of the markers to detect CAD at optimal cutoff values for PTGi, PTGVLFi, PTGr, and PTG CVD. In addition, correlation analyses between these markers and conventional autonomic nervous system and endothelial function markers were performed to understand the possible underlying physiological sources of the differences observed in marker values between CAD patients and healthy control patients. Additionally, $t$-tests were performed between two subgroups of the CAD patient group to determine whether diabetic or coronary artery bypass grafting (CABG) patients have significantly different PTGi marker values.

Results: Each spectral analysis PTG marker yielded a high specificity and sensitivity to detect CAD. Most notably, the PTG CVD score had a sensitivity of $82.5 \%$ and specificity of $96.8 \%$, at a cutoff of 2 , when used to detect $\mathrm{CAD}(P=0.0001$; area under the receiver operating characteristic curve $=0.967$ ). The PTG spectral analysis markers were well-correlated to other autonomic nervous system and endothelial function markers. CAD diabetic patients $(n=27)$ had a lower PTGi value compared with the CAD non-diabetic patients $(n=38)$ : and patients that underwent $\mathrm{CABG}(\mathrm{n}=18)$ had a higher PTGi value compared with the CAD without $\mathrm{CABG}$ surgery patients $(n=47)$. 
Conclusion: The spectral analysis of the photoplethysmography method is noninvasive, fast, operator-independent, and cost-effective, as only an oximeter and galvanic skin response device are required in order to assess in a single testing the autonomic nervous system and endothelial function. The spectral analysis techniques used on the photoplethysmogram, as outlined in this study, could be useful when used alongside conventional known cardiovascular disease risk markers.

Keywords: coronary artery disease, PTG spectral analysis, PTGi, PTGVLFi, PTGr, PTG CVD score

\section{Introduction}

Sudden cardiac death is the most likely consequence of both men $(50 \%)$ and women (64\%) with coronary artery disease (CAD). ${ }^{1}$ Currently, the only available strategy to reduce mortality in the at-risk population is primary prevention. ${ }^{1}$ CAD is a multifactorial disease that usually develops many years before any clinical symptoms are manifest; it is caused by risk factors such as hyperlipoproteinemia, high cholesterol diet, smoking, and diabetes mellitus. ${ }^{1}$

Over the last dozen years, appreciation of the role of endothelial dysfunction in CAD has burgeoned, ${ }^{2-4}$ as well as the role of cardiac autonomic neuropathy (CAN) in silent myocardial ischemia and cardiovascular morbidity. ${ }^{5}$

In addition, sympathetic stimulation significantly impairs the endothelial function by an alpha-adrenergic mechanism and therefore there is a relationship between the vascular and general homeostatic markers. ${ }^{6}$

Despite the fact that the autonomic nervous system and endothelial dysfunction increase cardiovascular risk, they do not contribute to either the Framingham score or the new American Heart Association/American College of Cardiology cardiovascular risk calculator. ${ }^{7}$

Endothelial dysfunction or damage is characterized by reduction of the bioavailability of nitric oxide, which upsets the balance between vasoconstriction and vasodilation and initiates a number of processes that promote hypertension. ${ }^{8}$ In addition, endothelial damage includes increased endothelial permeability, platelet aggregation, proinflammatory and procoagulatory states, and monocytes migration from the blood into the subendothelial intima and transformation into macrophages, which accumulate lipids to form the lipid core of atherosclerotic plaque. ${ }^{9}$

Plaque rupture can cause continued development of the atherosclerotic lesion by inducing further thrombus formation and release of more inflammatory mediators, resulting in continued luminal narrowing. ${ }^{10}$

A more drastic outcome of plaque rupture is arterial occlusion, which can result in myocardial infarction, ischemic stroke, or critical ischemia in peripheral tissues. ${ }^{11}$

The gold standard test for the evaluation of coronary endothelial function requires invasive quantitative coronary angiography to examine the changes in diameter in response to intracoronary infusions of endothelium-dependent vasodilators such as acetylcholine. ${ }^{12}$

Although several invasive tests have been employed successfully for the assessment of endothelial function over the last two decades, attention has now focused predominantly on noninvasive techniques.

A number of noninvasive techniques are now available for assessment of forearm vascular reactivity, and presently the most frequently used method involves flow-mediated dilation of the brachial artery, using ultrasound imaging. In this method, brachial diameter is measured at baseline and after an increase in arterial shear stress induced by inflation, then deflation, of a sphygmomanometric arm cuff that elicits reactive hyperemia and brachial vasodilation, predominantly caused by endothelium-derived nitric oxide release. ${ }^{13}$

One such newer methodology involves digital pulse amplitude tonometry, which measures volumetric changes in the fingertip using a probe that quantifies pulse amplitude in response to reactive hyperemia. ${ }^{14}$

Another device that is fairly quick and simple to use involves fingertip infrared light transmission photoplethysmography, which performs digital pulse volume waveform analysis and generates an automated reflection index (RI). ${ }^{15}$

CAN is also often underdiagnosed but can lead to severe morbidity and mortality, due to the associated cardiovascular burden. ${ }^{16}$ New evidence has emerged surrounding its complex pathways, but its full pathogenesis is yet to be understood. CAN manifests in a spectrum of subclinical and clinical presentations, ranging from resting tachycardia to cardiomyopathy.

Scintigraphy has enabled the diagnosis of CAN at a subclinical stage. ${ }^{17}$ Also, a battery of tests using tilt table and sudomotor evaluation induced by injection of acetylcholine are being used in diagnosis. ${ }^{18}$ The Toronto Consensus Panel on Diabetic Neuropathy has recently issued guidance for the diagnosis of CAN based on heart rate variability analysis and heart rate responses to deep breathing, standing, and Valsalva maneuver, as well as blood pressure response to standing. This guidance is considered as the gold standard in clinical testing for autonomic neuropathy. ${ }^{19}$

Despite the clinical need to assess endothelial function and the autonomic nervous system, several limitations have precluded its integration into clinical practice, partly owing to 
technical limitations that require extensive training, expensive equipment, and are labor-intensive, that have prompted a search for techniques inherently faster and easier to perform.

The TM-Oxi system assesses adrenergic and cardiovagal autonomic nervous system function in order to detect CAN as well as endothelial function in order to detect cardiovascular risk. It uses an automatic blood pressure device and an oximeter for heart rate detection and monitoring of blood volume and blood flow during the cardiac cycle (photoplethysmography). The SudoPath system assesses a patient's galvanic skin response by stimulating the postsympathetic cholinergic fibers in order to measure sweat gland density and skin microcirculation.

The objective of this study is to evaluate the TM-Oxi and SudoPath systems that use a patented, noninvasive analytical approach to assess new markers correlated to endothelial and autonomic nervous system functions by calculating the specificity and sensibility of these markers to detect CAD patients.

\section{Materials}

Two marketed medical devices, the TM-Oxi and SudoPath systems (LD Technology, Miami, FL, USA), were used in the study (see Figure 1).

The TM-Oxi uses a pulse oximeter and an automatic oscillometric blood pressure device managed by software. The pulse oximeter waveform or photoplethysmograph (PTG) is a simple and noninvasive optical technique that can be used to detect blood volume changes in the microvascular tissue bed. ${ }^{20}$

The PTG waveform is comprised of a pulsatile (alternating current) physiological waveform attributed to cardiac synchronous changes in the blood volume with each heartbeat and is superimposed on a slowly varying (direct current) baseline with various lower frequency components attributed to respiration, sympathetic nervous system activity, and thermoregulation. ${ }^{21}$

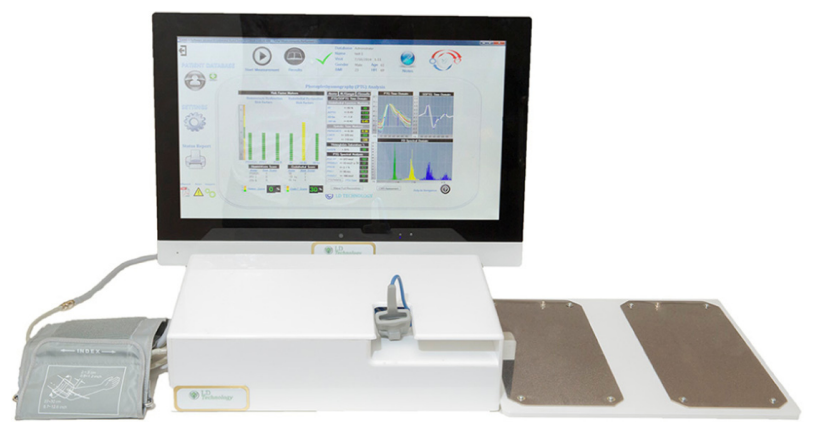

Figure I Photograph of the integrated medical devices (TM-Oxi and SudoPath).
The TM-Oxi performs PTG analysis of the beat-to-beat heart rate (RR intervals) and the PTG contour. The PTG analysis of RR intervals is based on the heart rate variability analysis as described by the Task Force of the European Society of Cardiology and the North American Society Task force, ${ }^{22}$ and from Ewing tests (eg, Valsalva maneuver, deep breathing, and change in posture) as described by the CAN subcommittee of the Toronto Consensus Panel on Diabetic Neuropathy. ${ }^{19}$

The PTG contour analysis is based on the PTG analysis as described in various studies in Asia, Europe, and the USA, ${ }^{23,24}$ and on the patented spectral analysis of the wave harmonics as described below. These measurements identify abnormal cardiovascular function, possibly stemming from autonomic nervous system and endothelial dysfunction. ${ }^{24}$

\section{The conventional PTG analysis performed by TM-Oxi}

The original PTG (Figure 2A) displays measurements of relative blood volume in the fingertip during cardiac events. The first derivative PTG (Figure 2B) peaks are used for accurate detection of the beat-to-beat heart rate ( $R R$ intervals). The second derivative PTG (Figure 2C) has been developed to allow for more accurate recognition of the inflection points on the original PTG wave. ${ }^{15}$ The ratios of the amplitude of $|a|$ to the amplitudes of $|b|,|c|,|d|$, and $|e|$ can aid in understanding arterial stiffness. Studies have shown that ratio negative second derivative $b / a$ and second derivative $d / a$ decrease in aging populations and some studies have linked these ratios directly to arterial distensibility. ${ }^{23,24}$ Additionally, the ratio between the heights of the dicrotic notch divided by the PTG peak is the Reflection Index (RI) ratio, which was also used in this study.

\section{SudoPath system: patented sudomotor function assessment (patent number \# US 61835064)}

The SudoPath is a galvanic skin response that uses two large stainless steel electrodes placed on the soles of the feet, where sweat gland density is very high. The device generates a low voltage signal with weak direct current that is fed to the active electrode. The current is delivered to the contralateral electrode-person circuit in two directions for each pathway. During the patented measurement process, the polarity is alternated at the middle of each measured pathway. The electrical stimulation provokes an electrochemical reaction on the bulk of the electrodes, measured as electro-conductance 

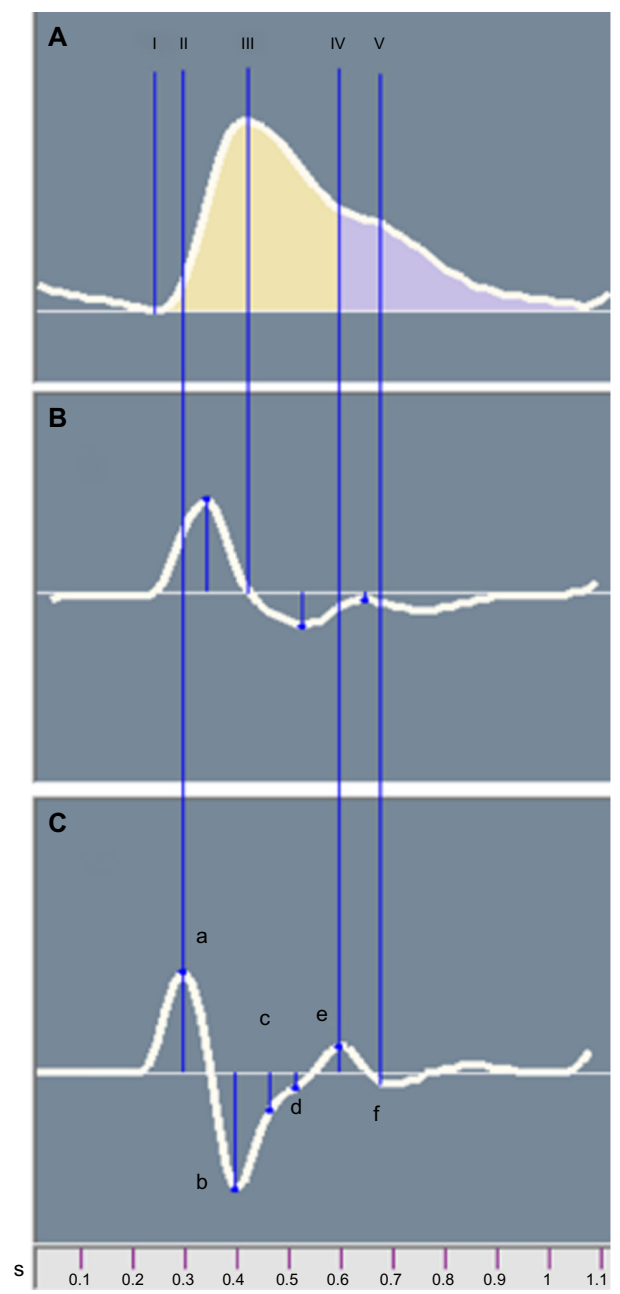

Figure 2 Averaged photoplethysmograph wave (A) and its first (B) and second (C) derivatives.

Notes: Points (I) to (V) represents the changes of the blood volume during the cardiac cycle. The yellow portion is the systolic time and the purple portion is the diastolic time. Points (a) to ( $f$ ) represents the projection of the points I to $V$ on the second derivative wave. response nitric oxide, which corresponds to the skin microcirculation, and electro-conductance response chloride, which corresponds to sweat gland density. The method is described on the manufacturer's web site (http://www.ldteck. $\underline{\mathrm{com} / \text { galvanic-skin-response.html). }}$.

\section{Patented signal processing analysis of the TM-Oxi (patent number PCT/IB20 I 3/002595)}

Analysis of the fast Fourier transform (FFT) of the PTG recording is a patented signal processing analytical method and could reveal information regarding autonomic nervous system activity and arterial status. In Figure 3, a 2-minute FFT PTG recording shows the characteristic peaks and frequency bands of a healthy subject. Average heart rate, power of each frequency band, peak amplitudes and their corresponding frequency bins, and total power (TP) can be extracted from this information. Harmonic components included in the FFT PTG include those associated with heart rate variability, the respiratory effects on the cardiac cycle, systolic and diastolic effects on peripheral blood flow, and the ability for blood vessels to dilate and contract. Harmonic components are very low frequency (PTGVLF), low frequency, and high frequency. In addition, parameters calculated from these components and from the SudoPath tests include: PTG index (PTGi), corresponding to the average of the amplitude of the three components expressed in volts per second (V/s), PTGVLF index (PTGVLFi), corresponding to the ratio of PTGVLF area/electro-conductance response nitric oxide expressed in milliseconds squared/microsiemens $\left(\mathrm{ms}^{2} / \mu \mathrm{S}\right)$, and PTG

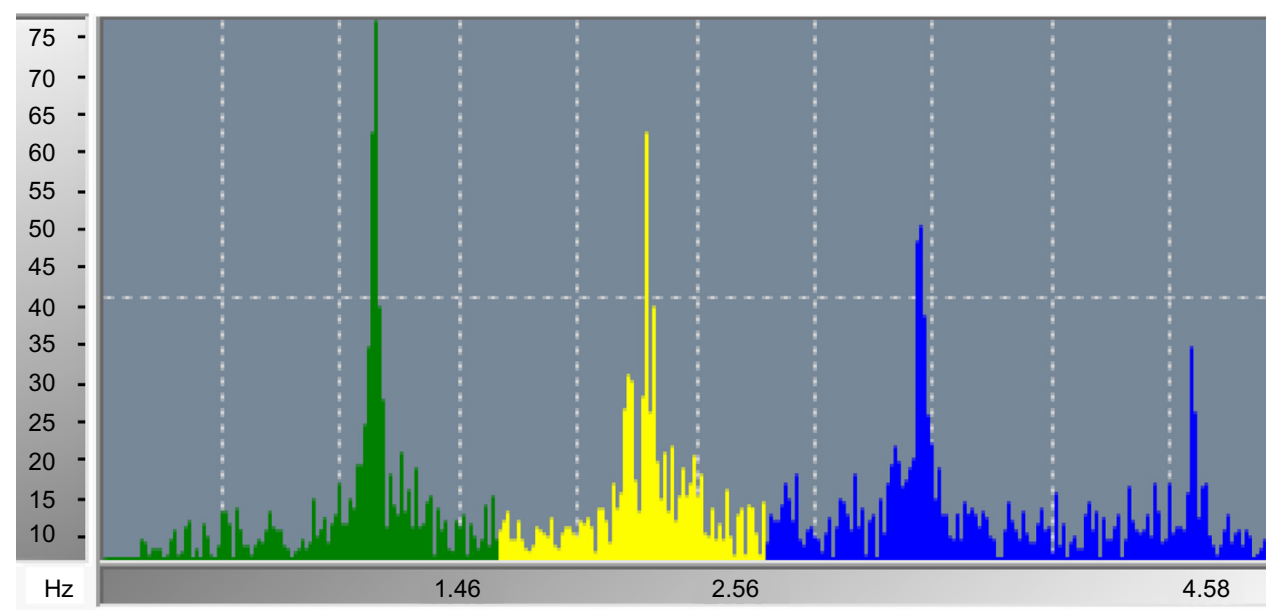

Figure 3 Spectral analysis of the first derivative of the photoplethysmography.

Notes: Green represents the very low frequencies (from 0 to $1.46 \mathrm{~Hz}$ ); yellow represents the low frequencies (from I.47 to $2.56 \mathrm{~Hz}$ ), and blue represents the high frequencies (from 2.57 to $5 \mathrm{~Hz}$ ). 
ratio (PTGr), corresponding to the PTGVLF area/PTGi ratio. A PTG cardiovascular (PTGCVD) score is calculated using the three markers PTGi, PTGVLFi, and PTGr. Each marker is scored as $0=$ normal, $1=$ borderline, and $2=$ abnormal, in order to calculate the PTGCVD score. Figure 4 shows the FFTPTG split into five groups by PTGi value.

\section{Experimental design}

Sixty-five patients ( 13 women) with a mean age of 62 years (range 40-80 years) who were diagnosed with CAD (group 1) and seventy-two subjects (29 women) with a mean age of 45.1 years (range 18-85 years) who claimed they were healthy (group 2) were included in the study.

Patients in group 1 were diagnosed with CAD by coronary angiography and all subjects underwent examination with the TM-Oxi and SudoPath systems at IPC Heart Care Centers in Mumbai, India. Table 1 describes the subject demographics and Table 2 describes the associated disease and treatment of CAD patients.

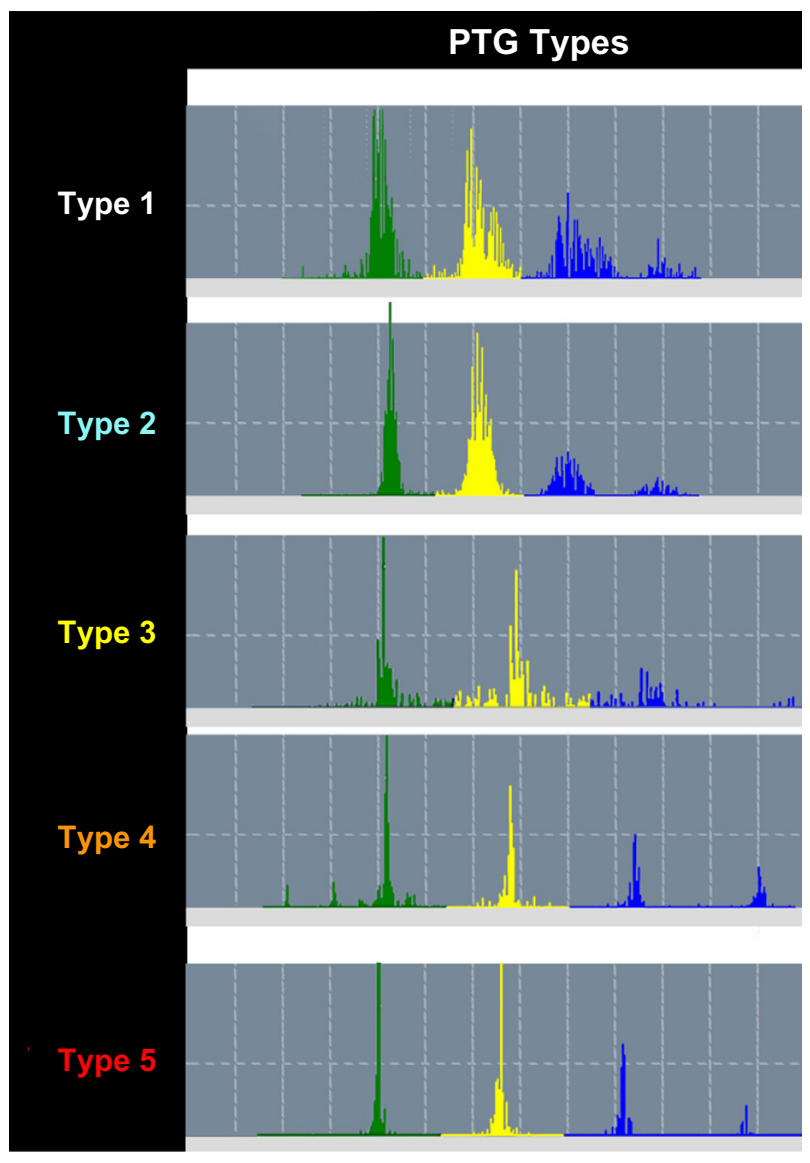

Figure 4 Spectral analysis of the photoplethysmography.

Notes: Classification by types according to the PTGi value. Type I: PTGi $\geq 60 \mathrm{~V} / \mathrm{s}$. Type 2: PTGi $\geq 40$ and $<60$ V/s. Type 3: PTGi $\geq 25$ and $<40$ V/s. Type 4: PTGi $\geq 15$ and $<25 \mathrm{~V} / \mathrm{s}$. Type 5: PTGi $<15 \mathrm{~V} / \mathrm{s}$.

Abbreviation: PTGi, photoplethysmography index.
Table I Subject demographic information

\begin{tabular}{lll}
\hline $\begin{array}{l}\text { General } \\
\text { demographic }\end{array}$ & Group I mean & Group 2 mean \\
\hline Men/women & $52 / 13$ & $43 / 29$ \\
Age (years) & 62.9 (range 40-80) & 45.1 (range I8-85) \\
$\begin{array}{l}\text { Body mass } \\
\text { index }\left(\mathrm{kg} / \mathrm{m}^{2}\right)\end{array}$ & 25.0 (range I6-35) & 24.1 (range I2.7-36.2) \\
$\begin{array}{l}\text { Systolic } \\
\text { pressure }(\mathrm{mmHg})\end{array}$ & 143 (range 103-202) & I27 (range 103-168) \\
$\begin{array}{l}\text { Diastolic } \\
\text { pressure }(\mathrm{mmHg})\end{array}$ & 82 (range 53-137) & 76 (range 55-10I) \\
\hline
\end{tabular}

The study was conducted according to the ethical principles of the Declaration of Helsinki. All of the subjects provided written informed consent and confidentiality was maintained for all subjects.

Subjects who were over the age of 18 years and had the ability to provide written informed consent were included in this study.

Patients were excluded from this study if they wore an automatic external defibrillator device, had erratic, accelerated, or mechanically-controlled irregular heart rhythms, had arterial fibrillation/flutter, had atrioventricular block, and/or had any implanted electronic device. These conditions were contraindications of the TM-Oxi and SudoPath devices.

\section{Data analysis}

Statistical analysis was performed using receiver operating characteristic curves to determine the specificity and sensitivity of PTGi, PTGVLFi, PTGr, and PTGCVD in detecting $\mathrm{CAD}$. The $t$-tests were also performed to determine if a significant difference in marker values between subgroups of group 1 (diabetic patients and patients who have had coronary artery bypass grafting $[\mathrm{CABG}]$ surgery), as outlined in this study, existed.

Table 2 Group I subject diseases and treatments

\begin{tabular}{ll}
\hline & Percentage \\
\hline Group I associated diseases & \\
Diabetes & $41 \%$ \\
Heart failure & $7 \%$ \\
Group I associated treatments & \\
Antihypertensive agents & $78 \%$ \\
Oral antidiabetic agents & $36 \%$ \\
Insulin treatment & $5 \%$ \\
Statins & $90 \%$ \\
Antiplatelet agents & $100 \%$ \\
CABG & $28 \%$ \\
PCI & $60 \%$ \\
\hline
\end{tabular}

Abbreviations: $\mathrm{CABG}$, coronary artery bypass grafting; $\mathrm{PCl}$, percutaneous coronary intervention or coronary angioplasty. 
Table 3 PTG spectral analysis markers results (data gathered from MedCalc statistical software) for detecting coronary artery disease

\begin{tabular}{llllll}
\hline $\begin{array}{l}\text { PTG spectral } \\
\text { analysis } \\
\text { markers }\end{array}$ & $\begin{array}{l}\text { Sensitivity } \\
\text { (\%) }\end{array}$ & $\begin{array}{l}\text { Specificity } \\
\text { (\%) }\end{array}$ & Cutoff & P-value & AUC \\
\hline PTGi & 86.1 & 87.3 & $\leq 40.8$ & 0.0001 & 0.926 \\
PTGVLFi & 86.1 & 93.6 & $>27$ & 0.0001 & 0.963 \\
PTGr & 73.8 & 93.6 & $>2$ & 0.0001 & 0.895 \\
PTGCVD score & 82.5 & 96.8 & $>2$ & 0.0001 & 0.967 \\
\hline
\end{tabular}

Abbreviations: AUC, area under the curve; PTG, photoplethysmography; PTGCVD, PTG cardiovascular disease; PTGi, PTG index; PTGr, PTG ratio; PTGVLFi, PTG very low frequency index.

Additionally, in order to understand the physiological parameters measured by PTGi, correlation analyses were performed between PTGi and RI, PTGi and heart rate variability score (TP), PTGi and age, and PTGi and CAN score.

\section{Sample size calculation and statistical analysis}

Based upon the preliminary study results, to have $90 \%$ power to detect a significant difference between subjects with the above mean and standard deviation at alpha $=0.05$, 30 subjects per group needed to be included. The sample size ( $\mathrm{n} \geq 30$ and $\alpha=0.05 \%$ ) was determined using MedCalc statistical software (http://www.medcalc.org/publications/ journals.php).

\section{Results}

MedCalc statistical software was used to perform the statistical analysis of the collected data with the function receiver operating characteristic curves in order to determine the specificity and sensitivity of the markers as shown in Table 3 and Figure 5.

MedCalc statistical software was used to perform the statistical analysis of the collected data with the coefficient of correlation in order to determine the correlation of the markers with endothelial and autonomic nervous system markers as shown in Table 4 and Figures 6-9.

The coefficient of correlation, $r$, was performed between the spectral analysis markers and RI (marker of endothelial function). As shown in Figure 6, $r=-0.87$ for PTGi/RI $(P=0.0001), r=0.76$ for PTGVLFi/RI $(P=0.0001)$, and $r=0.70$ for PTGr/RI $(P=0.0001)$.

The coefficient of correlation, $r$, was performed between the spectral analysis markers and TP (marker of heart rate variability score). As shown in Figure 7, $r=0.76$ for PTGi/TP

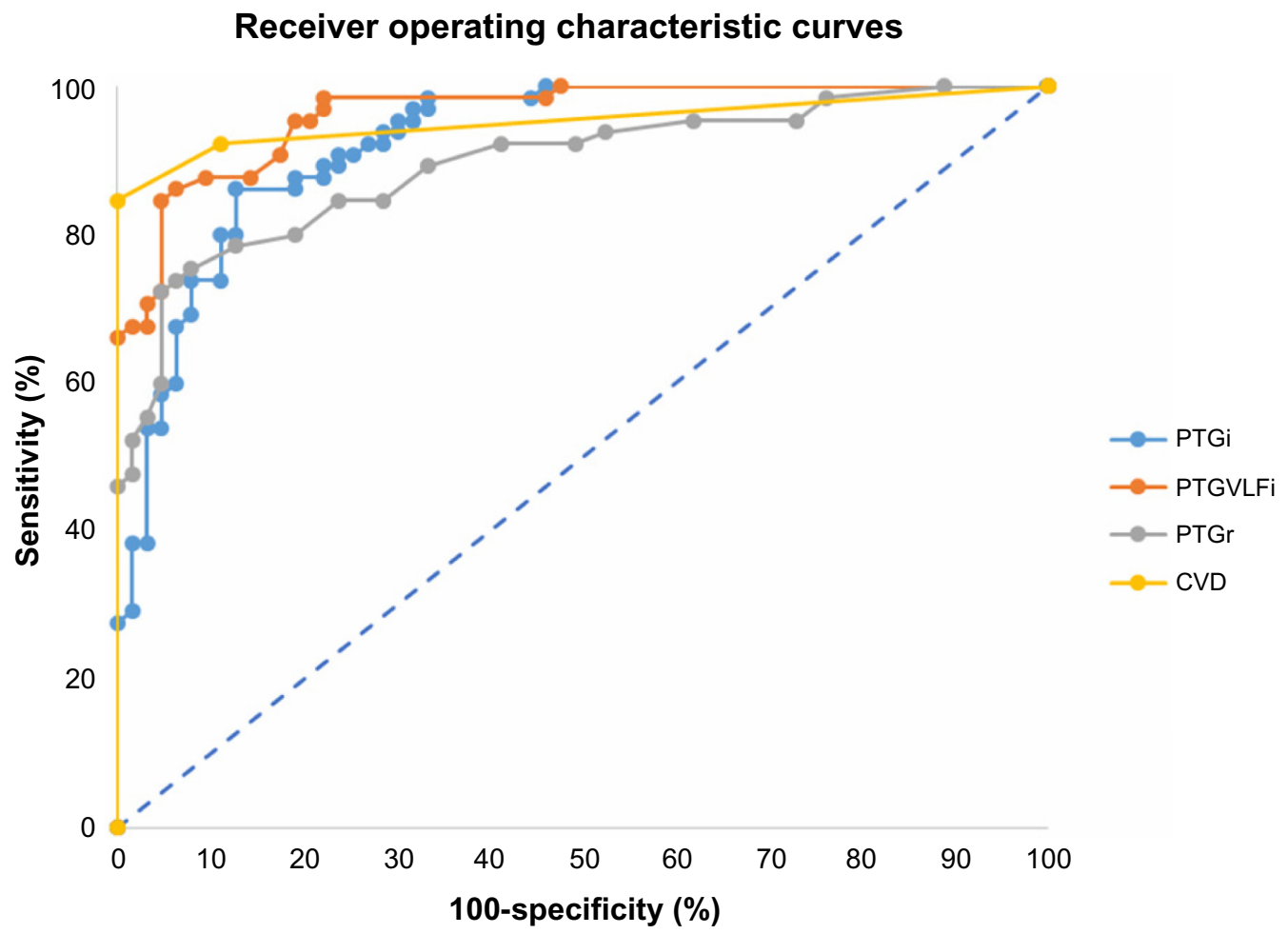

Figure 5 Receiver operating characteristic curves for photoplethysmography spectral analysis markers (image generated in Microsoft Excel from data gathered from MedCalc statistical software).

Abbreviations: CVD, cardiovascular disease; PTGi, photoplethysmography index; PTGr, photoplethysmography ratio; PTGVLFi, photoplethysmography very low frequency index. 
Table 4 Coefficient of correlation ( $r$ ) for PTG spectral analysis markers and endothelial/autonomic nervous system function markers (data gathered from MedCalc statistical software)

\begin{tabular}{lllll}
\hline $\begin{array}{l}\text { PTG spectral } \\
\text { analysis markers }\end{array}$ & RI & $\begin{array}{l}\text { HRV } \\
\text { TP }\end{array}$ & $\begin{array}{l}\text { CAN } \\
\text { score }\end{array}$ & Age \\
\hline PTGi coefficient $r$ & 0.87 & 0.76 & 0.56 & 0.54 \\
PTGVLFi coefficient $r$ & 0.76 & 0.51 & 0.64 & 0.47 \\
PTG $r$ coefficient $r$ & 0.70 & 0.50 & 0.41 & 0.32 \\
\hline
\end{tabular}

Abbreviations: CAN, cardiac autonomic neuropathy score; HRV TP, heart rate variability total power; PTG, photoplethysmography; RI, reflection index.

( $P=0.0001), r=-0.51$ for PTGVLFi/TP $(P=0.0001)$, and $r=0.50$ for $\mathrm{PTGr} / \mathrm{TP}(P=0.0001)$.

The coefficient of correlation, $r$, was performed between the spectral analysis markers and CAN score (marker of presence of cardiac autonomic neuropathy). As shown in Figure 8, $r=-0.56$ for PTGi/CAN $(P=0.0001), r=0.64$ for PTGVLFi/ $\mathrm{CAN}(P=0.0001)$, and $r=0.41$ for PTGr/CAN $(P=0.0001)$.

The coefficient of correlation, $r$, was performed between the spectral analysis markers and age. As shown in Figure 9, $r=-0.54$ for $\mathrm{PTGi} /$ age $(P=0.0001), r=0.47$ for $\mathrm{PTGVLFi} /$ age $(P=0.0001)$, and $r=0.32$ for PTGr/age $(P=0.002)$.

In addition, statistical analyses using $t$-tests were performed to compare PTGi values between the subgroups of the CAD patients.

CAD diabetic patients $(n=27)$ had a lower PTGi value compared to the CAD non-diabetic patients $(n=38)$. The mean PTGi in the CAD diabetic patient group was $24 \mathrm{~V} / \mathrm{s}$ versus $33 \mathrm{~V} / \mathrm{s}$ in the $\mathrm{CAD}$ non-diabetic patient group $(P=0.0001)$. Patients who underwent $\mathrm{CABG}(\mathrm{n}=18)$ had a higher PTGi value compared to that of the CAD patients who did not undergo CABG surgery $(n=47)$. The mean PTGi for CAD patients who had CABG surgery was $34 \mathrm{~V} / \mathrm{s}$

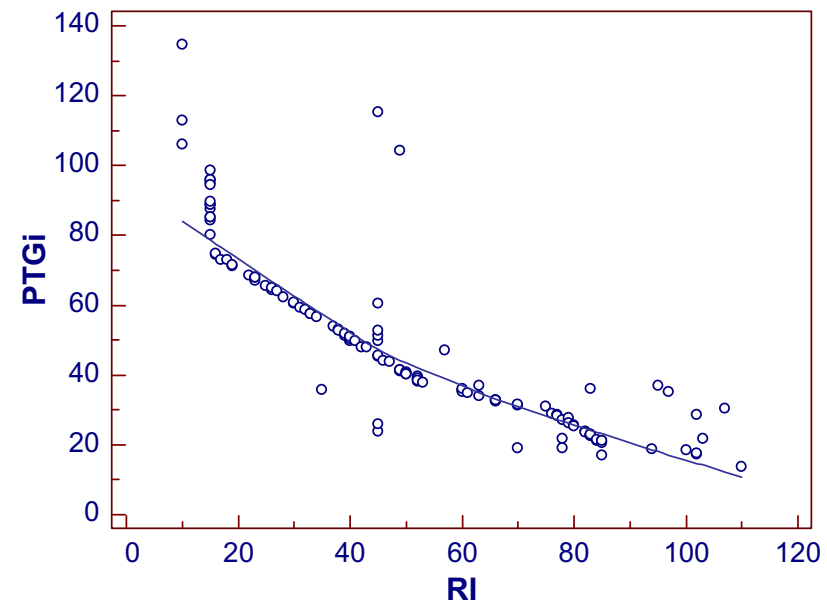

Figure 6 Scatter gram for evaluating the correlation of PTGi/RI. Abbreviations: PTGi, photoplethysmography index; RI, reflection index.

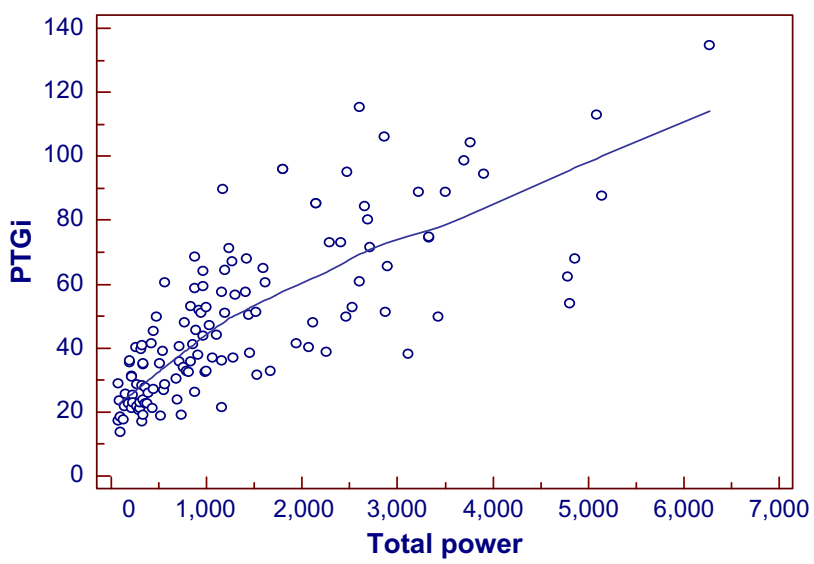

Figure 7 Scatter gram for evaluating the correlation of PTGi/total power. Abbreviation: PTGi, photoplethysmography index.

versus $30 \mathrm{~V} / \mathrm{s}$ for $\mathrm{CAD}$ patients who did not have $\mathrm{CABG}$ surgery $(P=0.01)$.

During the entirety of this study, no adverse events were reported with the use of the device.

\section{Discussion}

The results demonstrate that the PTG spectral analysis markers reflect homeostatic functions through correlation analyses with conventional markers of endothelial and autonomic nervous system function. The highest correlation of the PTG spectral analysis markers with the conventional markers of endothelial and autonomic nervous system functions are as follows:

PTGi correlated to endothelial function $(r=-0.87)$ and PTGVLFi correlated to CAN ( $r=0.64)$.

PTGr has a lower correlation coefficient with autonomic nervous system function than the previous markers, but still has a good correlation with endothelial function $(r=0.70)$.

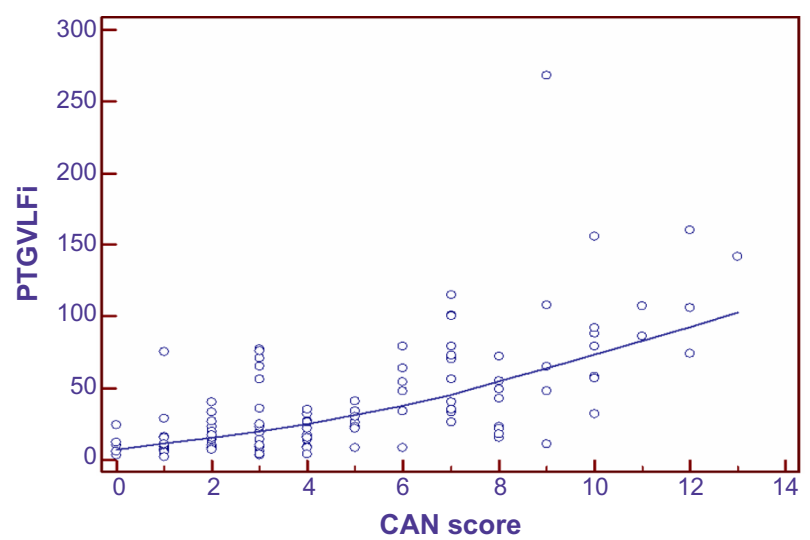

Figure 8 Scatter gram for evaluating the correlation of PTGVLFi/CAN score. Abbreviations: CAN, cardiac autonomic neuropathy; PTGVLFi, photoplethysmography very low frequency index. 


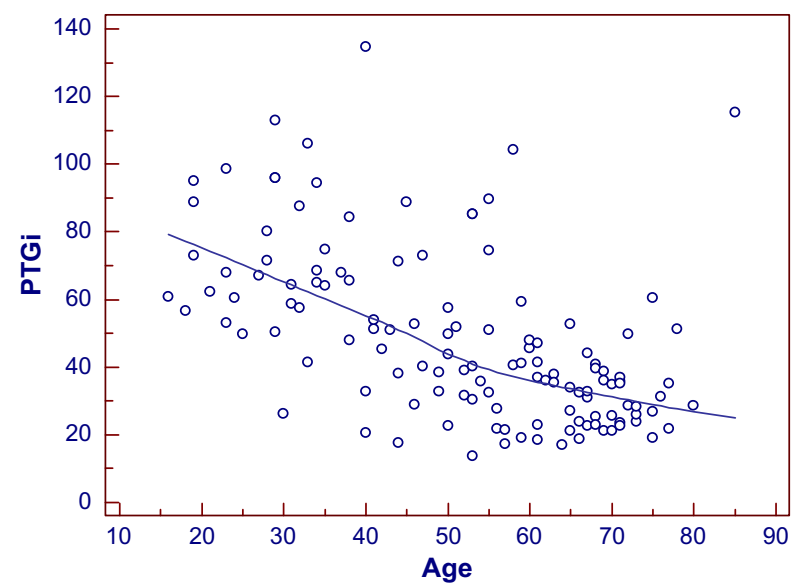

Figure 9 Scatter gram for evaluating the correlation of PTGi/age. Abbreviation: PTGi, photoplethysmography index.

Since the pathological state known as endothelial dysfunction is recognized as the main factor of the pathogenesis of $\mathrm{CAD}^{2}$ and $\mathrm{CAN}$ is a risk marker of mortality and cardiovascular morbidity, ${ }^{7}$ we can explain the good specificity and sensitivity of the PTG spectral analysis markers when comparing the CAD patient group to the healthy control group.

The digital pulse amplitude tonometry study showed that an EndoPAT index cutoff value of 1.67 yields a sensitivity of $82 \%$, a specificity of $77 \%$, and area under the curve of 0.82 when used to diagnose coronary endothelial function. ${ }^{14}$

The present study showed that PTGCVD score, based on spectral analysis PTG markers, with a cutoff value of 2, returns a roughly equivalent sensitivity $(82.5 \%)$ but higher specificity (96.8\%) and area under the curve (0.967).

Several limitations have precluded the endothelial and autonomic nervous system function assessment from being integrated into clinical practice, partly because the technical comprehension of these methods requires extensive training and expensive equipment, which has prompted a search for techniques that are inherently faster and easier to perform.

In the method outlined in this study, the PTG and sudomotor tests using tactile electrodes can be performed simultaneously, limiting the total time of the exam to 2 minutes. Additionally, these methods do not require operator intervention or invasive techniques, reducing overall patient discomfort.

Test results are immediately available from the software, including the normal range of the associated test results.

Although, the PTG spectral analysis markers provide only "surrogate" measures of endothelial and autonomic nervous systems, they are fast, comfortable, cost-effective, easy to interpret, and highly sensitive and specific when used to detect CAD. Therefore these methods could be easily integrated in daily practice and be very useful for the early detection of asymptomatic patients at high risk of arterial disease, autonomic neuropathy, and for CAD treatment follow-ups.

Finally, the PTG spectral analysis markers can be used alongside known conventional cardiovascular risk markers to strengthen the understanding of a patient's vascular state.

\section{Limitations}

In regard to the sample size of the population included in the present study, we recognize that the results need to be investigated further with more data from a larger cohort in a longitudinal study.

\section{Conclusion}

The spectral analysis of the photoplethysmography method is noninvasive, fast, operator-independent, and cost-effective, as only an oximeter and galvanic skin response device are required in order to assess in a single setting the autonomic nervous system and endothelial function. The spectral analysis techniques used on the photoplethysmogram, as outlined in this study, could be useful when used alongside known conventional cardiovascular disease risk markers.

\section{Acknowledgment}

The authors want to thank LD Technology for providing the medical systems that were used throughout the study.

\section{Disclosure}

The authors report no conflicts of interest in this work.

\section{References}

1. What is atherosclerosis? [webpage on the Internet]. Bethesda: National Heart, Lung, and Blood Institute; 2011. Available from: https://www. nhlbi.nih.gov/health/health-topics/topics/atherosclerosis/. Accessed September 24, 2014.

2. Widlansky ME, Gokce N, Keaney JF, Vita JA. The clinical implications of endothelial dysfunction. J Am Coll Cardiol. 2003;42(7):1149-1160.

3. Vita JA, Keaney JF Jr. Endothelial function: a barometer for cardiovascular risk? Circulation. 2002;106(6):640-642.

4. Deanfield JE, Halcox JP, Rabelink TJ. Endothelial function and dysfunction: testing and clinical relevance. Circulation. 2007;115(10): $1285-1295$.

5. Vinik AI, Ziegler D. Diabetic cardiovascular autonomic neuropathy. Circulation. 2007;115(3):387-397.

6. Jordan J, Tank J, Stoffels M, et al. Interaction between beta-adrenergic receptor stimulation and nitric oxide release on tissue perfusion and metabolism. J Clin Endocrinol Metab. 2001;86(6):2803-2810.

7. How accurate is the new AHA/ACC cardiovascular risk calculator? [webpage on the Internet]. JAMA Internal Medicine Blog; 2014. Available from: http://internalmedicineblog.jamainternalmed.com/2014/03/13/ how-accurate-is-the-new-ahaacc-cardiovascular-risk-calculator/. Accessed September 24, 2014. 
8. Schiffrin EL. A critical review of the role of endothelial factors in the pathogenesis of hypertension. J Cardiovasc Pharmacol. 2001;38 Suppl 2: S3-S6.

9. Bonetti PO, Lerman LO, Lerman A. Endothelial dysfunction: a marker of atherosclerotic risk. Arterioscler Thromb Vasc Biol. 2003; 23(2):168-175.

10. Anderson TJ, Gerhard MD, Meredith IT, et al. Systemic nature of endothelial dysfunction in atherosclerosis. Am J Cardiol. 1995;75(6): 71B-74B.

11. Behrendt D, Ganz P. Endothelial function: from vascular biology to clinical applications. Am J Cardiol. 2002;90(10C):40L-48L.

12. Kinlay S, Libby P, Ganz P. Endothelial function and coronary artery disease. Curr Opin Lipidol. 2001;12(4):383-389.

13. Corretti MC, Anderson TJ, Benjamin EJ, et al; International Brachial Artery Reactivity Task Force. Guidelines for the ultrasound assessment of endothelial-dependent flow-mediated vasodilation of the brachial artery: a report of the International Brachial Artery Reactivity Task Force. J Am Coll Cardiol. 2002;39(2):257-265.

14. Bonetti PO, Pumper GM, Higano ST, Holmes DR, Kuvin JT, Lerman A. Noninvasive identification of patients with early coronary atherosclerosis by assessment of digital reactive hyperemia. J Am Coll Cardiol. 2004;44(11):2137-2141

15. Millasseau SC, Kelly RP, Ritter JM, Chowienczyk PJ. Determination of age-related increases in large artery stiffness by digital pulse contour analysis. Clin Sci (Lond). 2002;103(4):371-377.

16. Kempler P, Tesfaye S, Chaturvedi N, et al; EURODIAB IDDM Complications Study Group. Autonomic neuropathy is associated with increased cardiovascular risk factors: the EURODIAB IDDM Complications Study. Diabet Med. 2002;19(11):900-909.
17. Dimitropoulos G, Tahrani AA, Stevens MJ. Cardiac autonomic neuropathy in patients with diabetes mellitus. World J Diabetes. 2014;5(1):17-39.

18. Autonomic neuropathy: Tests and diagnosis [webpage on the Internet]. Mayo Clinic; 2014. Available from: http://www.mayoclinic.org/ diseases-conditions/autonomic-neuropathy/basics/tests-diagnosis/ con-20029053. Accessed September 24, 2014.

19. Spallone V, Ziegler D, Freeman R, et al; on behalf of the Toronto Consensus Panel on Diabetic Neuropathy. Cardiovascular autonomic neuropathy in diabetes: clinical impact, assessment, diagnosis, and management. Diabetes Metab Res Rev. Epub June 22, 2011.

20. Allen J. Photoplethysmography and its application in clinical physiological measurement. Physiol Meas. 2007;28:R1-R39.

21. Foo JY, Lim CS. Dual-channel photoplethysmography to monitor local changes in vascular stiffness. J Clin Monit Comput. 2006;20(3) 221-227.

22. Heart rate variability: standards of measurement, physiological interpretation and clinical use. Task Force of the European Society of Cardiology and the North American Society of Pacing and Electrophysiology. Circulation. 1996;93(5):1043-1065.

23. Takazawa K, Tanaka N, Fujita M, et al. Assessment of vasoactive agents and vascular aging by the second derivative of photoplethysmogram waveform. Hypertension. 1998;32(2):365-370.

24. Nousou N, Urase S, Maniwa Y, Fujimura K, Fukui Y. Classification of acceleration plethysmogram using self-organizing map. Intelligent Signal Processing and Communications Symposium. 2006:681-684.
International Journal of General Medicine

\section{Publish your work in this journal}

The International Journal of General Medicine is an international, peer-reviewed open-access journal that focuses on general and internal medicine, pathogenesis, epidemiology, diagnosis, monitoring and treatment protocols. The journal is characterized by the rapid reporting of reviews, original research and clinical studies across all disease areas.

\section{Dovepress}

A key focus is the elucidation of disease processes and management protocols resulting in improved outcomes for the patient.The manuscript management system is completely online and includes a very quick and fair peer-review system. Visit http://www.dovepress.com/ testimonials.php to read real quotes from published authors. 